\title{
Accuracy-preserving Measurement Collection for Realistic Wireless Simulations
}

\author{
Mustafa Al-Bado \\ Cigdem Sengul \\ Ruben Merz \\ Telekom Innovation Laboratories at TU-Berlin \\ \{mustafa, cigdem\}@net.t-labs.tu-berlin.de, \{ruben.merz\}@telekom.de
}

\begin{abstract}
The wireless research community continuously questions the accuracy and the validity of wireless simulation models. While this led to a shift to testbeds for experimental evaluation, wireless testbeds only provide evaluations in a well-defined set of scenarios. Furthermore, even for the deployment of limited scenarios, testbeds require a complete system setup, which is a timeconsuming and challenging process. Hence, realistic simulation models are needed to get early performance results before going through the tedious work involved with testbed experimentation. In our prior work, we proposed the Berlin Open Wireless Lab (BOWL) Indoor Model (BIM) [1] to improve the accuracy of physical (PHY) layer simulation models. In this paper, we propose an optimization to the BIM model (OptBIM), which reduces the measurement complexity to build the model. We validate both BIM and OptBIM at the PHY and transport layers. We see that OptBIM shows similar performance to BIM at the PHY and transport layers. Also, the total number of measurements necessary was reduced by at least $18 \%$. The improvement comes from reducing the number of measurements by 8 times (e.g., from 144 to 18 in our study).
\end{abstract}

\section{INTRODUCTION}

Two approaches are typically used for wireless networking performance evaluation: testbeds and network simulators. On the one hand, wireless network testbeds allow for more realistic evaluations. On the other hand, their results are limited to a fixed testbed deployment and extremely hard to reproduce. Moreover, testbed development is a tedious and time-consuming process. Thanks to network simulators, these limitations can be overcome. In addition, network simulators can support the evaluation of richer and more varied scenarios than the limited setting of a testbed. However, wireless physical (PHY) layer models in packet-based simulators present a huge concern for simulation accuracy. Indeed, most simulators used in wireless research and industry [2], [3], [4], [5] typically support theoretical models for channel propagation. These models rely on idealistic assumptions about the environment and the communication conditions between transmitters and receivers. In [6], based on experiments in an outdoor network, it was shown that such idealistic assumptions greatly contradict the reality. Similarly, Roofnet testbed measurements [7], [8] also gave evidence that the assumptions of the theoretical models do not hold in a real wireless network.

Many studies have attempted to bridge the gap between simulation results and reality [9], [10], [11] by proposing measurement-based models. The common methodology followed by all is to characterize a specific environment and fit an empirical simulation model to the measurements. In [1], we proposed Berlin Open Wireless Lab (BOWL) Indoor Model (BIM), which includes a Frame Detection Ratio (FDR) model and a Frame Error Ratio (FER) model to represent the propagation and packet loss per-link in the BOWL indoor testbed. We evaluated BIM from a transport layer perspective and showed a significantly better accuracy in comparison to other simulation models, including measurement-based models [12]. However, BIM requires extensive active measurements of all links at all data-rates to represent the propagation and packet loss characteristics efficiently on a particular channel.

This work focuses on decreasing the complexity of running measurements for BIM without affecting its accuracy. The main contribution of our work is to exploit the relationship between the lowest data-rate and the higher data-rates. This relationship helps reducing the number of measurements for the rate characterization measurements (see Section V) by a factor of eight (i.e. from 144 to 18 measurements in the BOWL indoor testbed). Decreasing the number of measurements also reduces the time-variance effects in our measurements seen at different data-rates. The evaluations at both the PHY and transport layers indeed show that OptBIM achieves similar or better accuracy compared to BIM.

The rest of the paper is organized as follows. We present the related work in Section II. Section III describes the BOWL testbed and our measurement and simulation setup for OptBIM. In Section IV, we describe BIM. Section V describes the required measurements and the simulation implementation of OptBIM. In Section VI, we evaluate the OptBIM accuracy at the PHY and transport layers. Finally, we conclude in Section VII.

\section{RELATED WORK}

The accuracy of wireless models in packet-based simulators has been often questioned by the wireless research community [13], [14], [15], [16]. Well-known network simulators [2], [3], [4], [5] support path-loss models such as the Friis free-space model, the two-ray ground model and various shadowing models. The Friis model takes into account the distance between the two communication edges and the medium density to calculate signal attenuation. The two-ray ground model considers the direct and ground reflection in addition to the parameters of Friis model. Shadowing models add a stochastic component to take into account signal fading. 

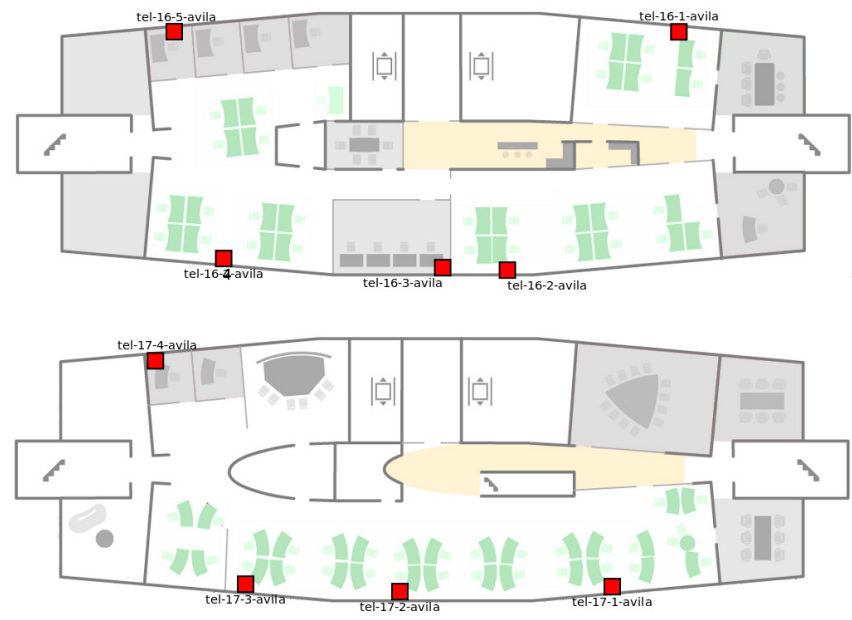

Fig. 1. The BOWL indoor testbed spans two floors. There are five nodes on the 16th floor (top picture) and four nodes on the 17th floor (bottom picture) The host names are indicated with the convention: tel-floor-node.

These models are shown to not capture propagation in a real environment accurately. Therefore, several works attempted to increase the credibility of wireless simulation results by using measurement-based models at the PHY layer, for instance, for mobility propagation and channel deferral [10]. In [9], [10], the authors used measurement results from a rural area to feed a distance-based models. Nevertheless, their results are not applicable to indoor environments where obstacles and artifacts significantly impact signal propagation and even lead to packet loss. In [11], two PHY layer models were proposed based on two measurement approaches in an indoor network. The first approach relied on testbed measurements, where each node in the testbed took the role of the transmitter while the others sniffed and recorded the receive signal strength (RSS) of the received packets. In the second approach, just a pair of nodes were placed in arbitrary places in the building. At each of these places, one of the nodes took the role of the transmitter while the other acted as the receiver. Both measurement studies were used to feed a log-distance shadowing model. However, several other studies showed that there is no direct relationship between RSS and the distance between the transmitter and the receiver in an indoor environment and more representative models are needed [1], [17]. In this paper, we present the Optimized Bowl Indoor Model (OptBIM) which takes a step in the direction of more efficient and accurate simulation models.

\section{BOWL Testbed And Measurement Setup}

Our simulation models are based on the measurements carried out in BOWL indoor testbed [18] at Telekom Innovation Laboratories, in Berlin, Germany. In this section, we first describe our testbed and then, explain the measurement and simulation setup used in this work.

\section{A. System Description}

The BOWL indoor testbed consists currently of nine nodes, five of them are deployed in one floor, and the remaining four reside on the floor above (see Figure 1). The host names indicate the floor and the node numbers. For instance, tel-16-2 is the second node on the 16th floor. Each node has a Gatework Avila GW2348-4 platform with 64 Mbyte of RAM, an Intel XScale IXP425 $533 \mathrm{MHz}$ processor (ARM architecture) and two Wistron CM9 miniPCI IEEE 802.11abg wireless network interface cards (NIC), and a $8 \mathrm{dBi}$ gain omnidirectional antenna (with a $2 \mathrm{~dB}$ loss because of cabling). The wireless NIC is an Atheros AR5213A [19]. We use OpenWrt 8.09.2 with Linux kernel 2.6.26.8 as the operating system. The wireless driver is the version maintained by OpenWrt, with revision number 3314 with HAL 20090508. Additionally, all nodes have a dedicated $100 \mathrm{Mbit} / \mathrm{s}$ Ethernet management interface, which is used to collect measurement results on a central server. To generate experiment traffic, we use one dedicated load generator machine (loadgen) with Intel(R) $2.80 \mathrm{GHz}$ processor, $4 \mathrm{CPU}$ cores and $6 \mathrm{~GB}$ RAM. The operating system is Linux version 2.6.32.

\section{B. Measurement Setup}

In all experiments, we used only one of the available wireless interfaces. The interface was in one of these following modes: ahdemo ${ }^{1}$ or monitor ${ }^{2}$ mode. The ahdemo mode was used when the node was transmitting data. In this mode, nodes do not transmit any management packets (e.g., beacons), which allows more controlled experiments. The monitor mode was used to capture packets. This mode uses the socalled radiot $\mathrm{pp}^{3}$ header, which includes some PHY parameters such as RSS. The packet size in all experiments was $1024 \mathrm{~B}$. The underlying physical layer was IEEE 802.11a [20] and we used channel 44 because it was the channel with the lowest interference in our building at the time of running the experiments. The adaptive noise immunity and weak signal detection mechanisms were enabled [21], [22]. We use two scenarios to build and evaluate our models:

- Broadcast scenario: One transmitter broadcast UDP datagrams using the iper $\mathrm{f}^{4}$ traffic generator while the other nodes attempt to receive the transmitted packets. Here, the transmitter pushes to saturate the medium to obtain more accurate information about the channel state.

- Unicast scenario: TCP or UDP traffic is sent between a pair of nodes. Fig. 2 shows the setup and the traffic direction for a unicast session between two chosen nodes. To ensure a consistent network stack between the experiment and simulation, the traffic is generated by the ns- 3 application script on the loadgen, which forwards it to the transmitter. Then the transmitter sends the traffic to the receiver via the wireless medium. Finally, the traffic is forwarded back to the loadgen. In case of TCP, acknowledgement packets use the opposite direction. We use the EmuNetDevice module to associate ns-3 with the real network. For TCP traffic, we use TcpReno module with

\footnotetext{
${ }^{1}$ http://madwifi-project.org/wiki/UserDocs/AhdemoInterface

${ }^{2} \mathrm{http} / / /$ madwifi-project.org/wiki/UserDocs/MonitorModeInterface

${ }^{3}$ http://madwifi-project.org/wiki/DevDocs/RadiotapHeader

${ }^{4}$ http://iperf.fr/download/iperf_2.0.2/doc/index.html
} 


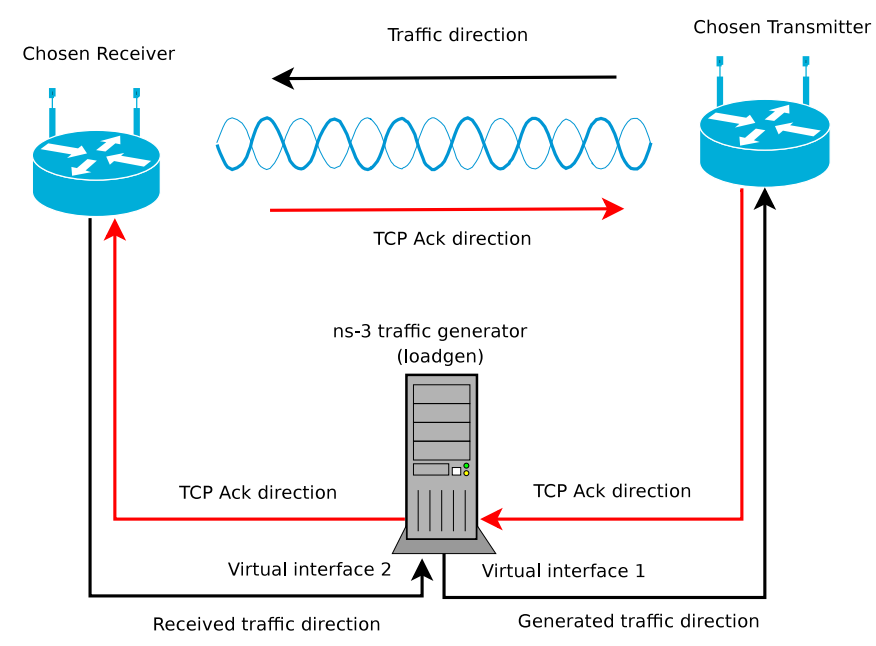

Fig. 2. The setup for a unicast session between two nodes.

1024 Bytes (B) segment size. The OnOff Application module is used to generate the traffic with $15 \mathrm{Mbps}$ for TCP and 20 Mbps for UDP. This is a result of CPU constraints, which does not allow for higher throughput.

\section{Simulation Setup}

For the simulation study, we use ns- 3 version $3.10^{5}$. The simulated topology represents the BOWL indoor testbed as shown in Fig. 1. The simulation setup follows the measurements. In particular, for broadcast scenario traffic, we use CBR UDP with 1024 B datagram size. The setup of this scenario matches the iperf traffic generator setup as well. For unicast traffic scenarios, we use the same settings as the measurements. The simulation results are presented with their $95 \%$ confidence intervals from 10 simulation runs.

\section{BOWL INDOOR MODEL (BIM)}

Our main simulation model, the BOWL indoor model (BIM) [23], was originally proposed in [1]. It is a measurement-based model of the BOWL indoor network and comprises a radio propagation model, a frame detection ratio (FDR) model and a frame error ratio (FER) model. FDR is the ratio of all the detected frames (i.e., includes the frames with errors) to the transmitted packets. FER is the ratio of frames with errors to FDR. A frame has an error when it does not pass the Cyclic Redundancy Check (CRC).

Our model is based on per-link RSS distributions. Based on our measurements (see Section III-B), for each link and for each combination of data-rate and channel frequency, we build an empirical cumulative distribution function (ECDF) of the RSS. Our data includes only the RSS of detected frames (i.e., excludes frames that were dropped by the radio signal detection unit). However, as we know exactly how many frames were transmitted, and therefore, for each lost frame, we represent its RSS as the corresponding FDR threshold (i.e., the noise floor plus a data-rate specific correction factor, see [1]

\footnotetext{
${ }^{5}$ The latest release 3.13 does not contain any changes that affect our work.
}

for more details). In the simulator, the RSS value for a given frame is obtained simply by sampling the RSS distribution of the corresponding link for the given data-rate and channel. The frames with RSS below FDR threshold are dropped. Frames equal to or higher than the threshold are passed to the FER model, where the RSS and data-rate of the frame is used to look up the corresponding frame error probability $p$. This probability is then compared to a uniformly sampled random variable $q(0 \leq q \leq 1)$. If $q>p$, the frame is successfully received. Else, the frame contains an error and is dropped.

BIM relies on two types of measurements to build its models: (1) rate characterization measurements, which serve to characterize the desired network environment in the simulator (e.g., night and office hours represent different environments) and (2) FER measurements, which are used to derived the FER database. In the next section, we will discuss these measurements in more detail in the context of OptBIM, explaining in detail the similarities and differences with BIM.

Finally, BIM is implemented in ns-3 as a new propagation model. It makes the following modifications to the YansWifiphy class:

- Support for feeding measurement-based RSS distributions

- Per-rate FDR threshold

- A FER model with RSS and modulation as parameters

- Transmit power behavior of Atheros hardware

- Recording of dropped frames in the radiotap trace

\section{OPTIMIZED BIM (OPTBIM)}

As mentioned in the previous section, BIM relies on active measurements of all nodes for each data-rate and channel. In this section, we explain how OptBIM is able to reduce the number of measurements and maintain similar accuracy to BIM.

\section{A. Building Models from Measurements}

OptBIM, similar to BIM, relies on both rate characterization and FER measurements. However, it significantly reduces the number of measurements necessary for rate characterization. OptBIM takes advantage of our finding in prior work [1], which confirms that changing the data-rate at the transmitter should not affect the RSS range at the receiver. However, the changes in data-rate have a major impact on FDR at the receiver. Therefore, in this paper we propose an empirical model to predict the RSS and FDR of the higher data-rates based on the measurements of the lowest data-rate (i.e, $6 \mathrm{Mbps}$ for IEEE 802.11a). This reduces the number of experiments by 8 (as we do not need to run measurements for each of the 8 data rates). Moreover, unstable environment conditions (e.g, during the office hours) may cause inconsistency in RSS ranges observed for different data-rates. In other words, in a dynamic environment different RSS ranges may be observed for different data rates because of changing channel conditions (e.g., due to moving objects). Hence, by reducing the number and hence, the time of running the rate characterization measurements, we also expect to alleviate loss of accuracy in multi-rate scenarios. 
Hence, OptBIM is similar to BIM and requires two measurement setups. However, the rate characterization measurements in BIM are only conducted for the lowest data-rate. In these measurements, the broadcast scenario (see Section III-B) is used with UDP datagrams of $1024 \mathrm{~B}$ at lowest data-rate and one transmit power (in our case $6 \mathrm{Mbps}$ and $13 \mathrm{dBm}$ ). The duration of the experiment and number of repetitions depend on the environment to be characterized. For instance, dynamic environments need higher number of repetitions than stable environments to capture the changes in the environment.

OptBIM uses the same setup as BIM for FER measurements. For OptBIM, these measurements need to be run in a stable environment where object mobility is low and the interference is at its lowest level. Using this setup, we build the FER database and an FDR look-up table that decide the FDR of the higher data-rates by using the ECDF distribution of RSS of the lowest data-rate. The FER database and the FDR look-up table is built in two steps:

- We run the broadcast scenario with 9 nodes using UDP datagrams of $1024 \mathrm{~B}$ at 8 data-rates (i.e, all 802.11a datarates) and 8 transmission powers (i.e, $0,2,4,6,8,10,12$ and $13 \mathrm{dBm})$. In other words, we run $9 \times 8 \times 8=576$ measurements. Each measurement runs for 180 seconds. We use different transmission powers to obtain finer granularity in the measured RSS ranges. This allows to cover more RSS values in the measurements and not to resort to interpolation for missing RSS values. Even though it is not always necessary, we repeated this experiment twice to make sure the environment is stable by comparing the difference between measured RSS ranges in both repetitions. Hence, we gained confidence on the FDR look-up tables, which establish the relationship between the low data-rate and high data-rate FDR values.

- We next aggregate measurements assuming that all nodes have the same FDR value for a particular RSS. Earlier, we have shown that aggregating data this way does not impact accuracy at higher network layers [12]. Hence, the FDR look-up table is built based on the assumption that the FDR value for a given data-rate is fixed for a certain RSS value. Table I shows an example of the FDR look-up table for the very low RSS values. Consider the case for 24 Mbps. For each RSS value, there are associated real and normalized FDR values. The real column represents the actual measured FDR (e.g., $21.12 \%$ for -90 RSS)). The corresponding norm. column represents the real FDR values normalized to the FDR of the lowest datarate (e.g., $21.12 / 88=24 \%$ ). We use this normalization because, in simulation, OptBIM uses the ECDF database for the lowest data-rate to draw the RSS value for a given packet. Then this RSS value and the data-rate of the packet are used to look up the corresponding FDR value. Since the ECDF database is biased with the lowest datarate FDR, this bias should be removed by normalization.

In summary, in our testbed, both BIM and OptBIM use 576 FER measurement runs. But OptBIM reduces the number of rate characterization measurements for a given environment by 8 . In our study, BIM needed 144 measurements, while OptBIM required only 18 . Note that as more environments need to modeled (e.g., the same space during high mobility), the number of rate characterization measurements also need to increase for both BIM and OptBIM. For modeling only one environment, OptBIM reduces the total number of measurements by $\approx 18 \%$.

\section{B. OptBIM in the Simulator}

As in [1], two FER models are used: default and networkbased models. Both models are fed by the ECDF distribution for the lowest data-rate.

- In the default model, the drawn RSS value is pushed to the default ns-3 FER model. The default model does not use the FDR look-up table because we assume that the default FER model in ns-3 was designed to process the RSS values of received packets without pre-handling.

- In network-based model, after picking up the new RSS value from the lowest data-rate ECDF database, the RSS value is used to find the proper $\operatorname{FDR}(q)$ from the lookup table. Then, we pick a random number $(p)$. if $(q>p)$ then the packet is passed to the measurement-based FER model in BIM.

Table II summarizes the differences between BIM and OptBIM.

\section{Vi. Evaluation}

In this section, we evaluate OptBIM at the PHY and transport layers against real measurements and also in comparison to BIM. The aim of our evaluation is to show whether OptBIM can represent the medium in an accuracy similar or close to the original BIM. The evaluations is run on channel 44 and at a transmission power of $12 \mathrm{dBm}$.

\section{A. PHY Layer Evaluation}

In this case, the broadcast scenario is used (see III-B). Each node transmits at three data-rates: 6, 24 and 54Mbps continuously. The OptBIM model uses the ECDF database of $6 \mathrm{Mbps}$ and the FDR look-up table (see section $\mathrm{V}$ ) to produce 24 and $54 \mathrm{Mbps}$ data packets. In contrast, BIM uses, in addition, the ECDF from 24 and $54 \mathrm{Mbps}$ to produce the data packets RSS values for these data-rates.

Figures 3(a) and 3(b) show the Frame Reception Ratio (FRR) for all the potential links in the BOWL indoor testbed for 24 and 54 Mbps data-rates. The FRR is defined as the proportion of correctly received frames by the receiver to the number of transmitted frames from the sender. Both figures show that OptBIM has similar accuracy to BIM. Furthermore, the measurement-based FER models provide more accuracy compared to the ns-3 default FER model.

\section{B. Transport Layer}

For transport layer validation, we use the unicast scenario for both TCP and UDP traffic for all theoretical links in the BOWL indoor testbed. We assumed that we have a fully 
TABLE I

REAL AND NORMALIZED FDR VALUES FOR LOW RSS FOR ALL 802.11A DATA-RATES IN BOWL INDOOR TESTBED (THE RESULTS ARE IN \%)

\begin{tabular}{|c|c|c|c|c|c|c|c|c|c|c|c|c|c|c|c|c|}
\hline \multirow[t]{2}{*}{ RSS } & \multicolumn{2}{|c|}{$6 \mathrm{Mbps}$} & \multicolumn{2}{|c|}{9 Mbps } & \multicolumn{2}{|c|}{$12 \mathrm{Mbps}$} & \multicolumn{2}{|c|}{$18 \mathrm{Mbps}$} & \multicolumn{2}{|c|}{$24 \mathrm{Mbps}$} & \multicolumn{2}{|c|}{$36 \mathrm{Mbps}$} & \multicolumn{2}{|c|}{$48 \mathrm{Mbps}$} & \multicolumn{2}{|c|}{$54 \mathrm{Mbps}$} \\
\hline & real & norm. & real & norm. & real & norm. & real & norm. & real & norm. & real & norm. & real & norm. & real & norm. \\
\hline-95 & 0.01 & 2100 & $\overline{0}$ & $\overline{0}$ & $\overline{0}$ & $\overline{0}$ & $\overline{0}$ & $\overline{0}$ & $\overline{0}$ & $\overline{0}$ & $\overline{0}$ & $\overline{0}$ & $\overline{0}$ & $\overline{0}$ & $\overline{0}$ & $\overline{0}$ \\
\hline-94 & 2 & 100 & 0 & 0 & 0 & 0 & 0 & 0 & 0 & 0 & 0 & 0 & 0 & 0 & 0 & 0 \\
\hline-93 & 9 & 100 & 4.05 & 45 & 1.26 & 14 & 0.45 & 5 & 0 & 0 & 0 & 0 & 0 & 0 & 0 & 0 \\
\hline-92 & 41 & 100 & 22.55 & 55 & 16.4 & 40 & 4.51 & 11 & 0.41 & 1 & 0 & 0 & 0 & 0 & 0 & 0 \\
\hline-91 & 76 & 100 & 72.2 & 95 & 68.4 & 90 & 45.6 & 60 & 12.92 & 17 & 0.76 & 1 & 0 & 0 & 0 & 0 \\
\hline-90 & 88 & 100 & 88 & 100 & 83.6 & 95 & 57.2 & 65 & 21.12 & 24 & 2.64 & 3 & 0 & 0 & 0 & 0 \\
\hline-89 & 95 & 100 & 95 & 100 & 95 & 100 & 76 & 80 & 38 & 40 & 4.75 & 5 & 0 & 0 & 0 & 0 \\
\hline-88 & 98 & 100 & 98 & 100 & 98 & 100 & 83.3 & 85 & 63.7 & 65 & 9.8 & 10 & 0.98 & 1 & 0 & 0 \\
\hline-87 & 100 & 100 & 100 & 100 & 100 & 100 & 100 & 100 & 92 & 92 & 25 & 25 & 5 & 5 & 1 & 1 \\
\hline-86 & 100 & 100 & 100 & 100 & 100 & 100 & 100 & 100 & 94 & 94 & 35 & 35 & 5 & 5 & 1 & 1 \\
\hline
\end{tabular}

TABLE II

THE UNDERLYING OF BIM AND OPTBIM VERSIONS

\begin{tabular}{|l|l|l|l|l|l|l|}
\hline Model version & $\begin{array}{l}\text { RSS from ECDF distribution } \\
\text { for each data-rate }\end{array}$ & $\begin{array}{l}\text { RSS from the lowest data- } \\
\text { rate ECDF distribution }\end{array}$ & $\begin{array}{l}\text { FDR } \\
\text { threshold }\end{array}$ & $\begin{array}{l}\text { FDR look-up } \\
\text { table }\end{array}$ & $\begin{array}{l}\text { default } \\
\text { ns-3 FER }\end{array}$ & $\begin{array}{l}\text { network-based } \\
\text { FER }\end{array}$ \\
\hline \hline BIM network-based & $\mathrm{X}$ & & $\mathrm{X}$ & & & \\
\hline BIM default & $\mathrm{X}$ & & & & $\mathrm{X}$ & $\mathrm{X}$ \\
\hline OptBIM network-based & & $\mathrm{X}$ & & $\mathrm{X}$ & & \\
\hline OptBIM default & & $\mathrm{X}$ & & & $\mathrm{X}$ & \\
\hline
\end{tabular}

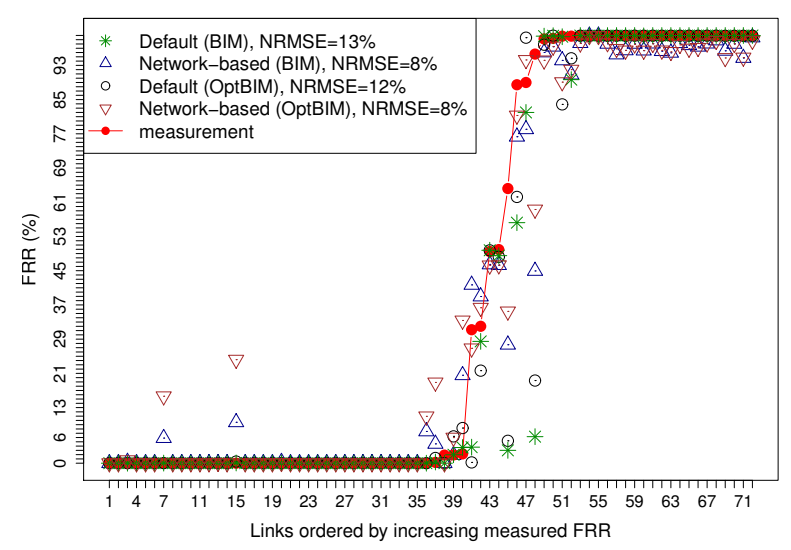

(a) $24 \mathrm{Mbps}$

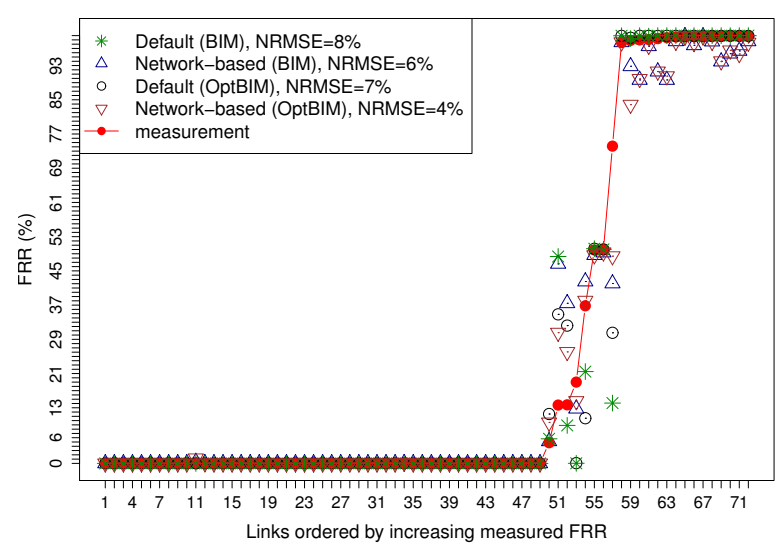

(b) $54 \mathrm{Mbps}$

Fig. 3. FER validation for two different data-rates. connected mesh because during measurements the presence of certain links fluctuate. The measurements for both models are run based on the broadcast scenario. The broadcast scenario is run before and after the unicast scenario to capture the channel status as best as possible during the unicast communication (i.e., TCP and UDP traffic). For $24 \mathrm{Mbps}$ test, we run the broadcast scenario for 6 and $24 \mathrm{Mbps}$ from both sides of the connection. For $54 \mathrm{Mbps}$ test, we run the broadcast scenario for 6,24 and $54 \mathrm{Mbps}$ from both sides of the connection. We also evaluate $24 \mathrm{Mbps}$, because the Ack for $54 \mathrm{Mbps}$ is sent at $24 \mathrm{Mbps}$.

Figures 4(a) and 4(b) show that both models have similar root mean square error (RMSE): $15-16 \%$ for UDP and 11$12 \%$ for TCP. The same observation also holds for $54 \mathrm{Mbps}$ results in Figures 5(a) and 5(b): 3-5\% for UDP and 8-9\% for TCP.

\section{CONCLUSION}

In this paper, we develop an optimization to our sitespecific indoor simulation model, BIM, which uses the ECDF distribution of RSS for the lowest data-rate to represent all data-rates. We evaluated two versions of OptBIM: the default version utilizes the analytical FER model in ns-3 while the network-based version uses an empirical model built to fit with BOWL testbed conditions for FER model [1]. The results from the PHY and transport layer evaluations show that OptBIM performs similar to BIM while it significantly reduces the number of measurements necessary to build the model. For future work, we plan to build a site-specific model for BOWL outdoor testbed. Moreover, we plan to investigate the accuracy of BIM in a multi-hop scenarios.

\section{REFERENCES}

[1] M. Al-Bado, R. Merz, C. Sengul, and A. Feldmann, "A site-specific indoor link model for realistic wireless network simulations," ser. SIMUTOOLS, March 2011. 


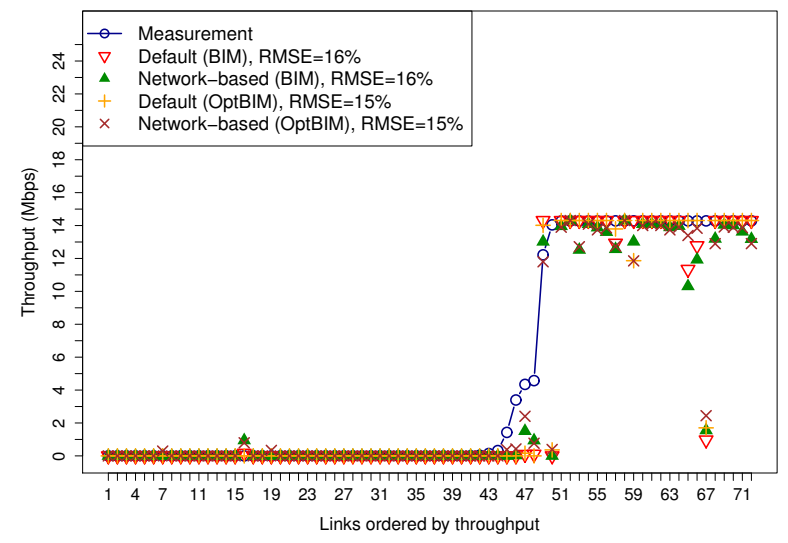

(a) UDP 24 Mbps

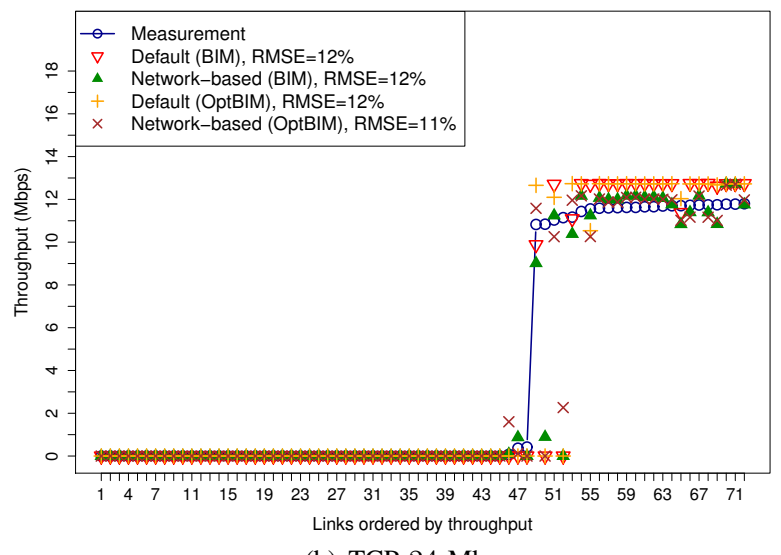

(b) TCP $24 \mathrm{Mbps}$

Fig. 4. Single flow traffic validation for $24 \mathrm{Mbps}$ data-rate.

[2] “ns-2," http://www.isi.edu/nsnam/ns/ns-documentation.html.

[3] "Opnet," http://www.opnet.com/.

[4] "Qualnet," http://www.scalable-networks.com/products/qualnet/.

[5] "ns-3," http://www.nsnam.org/.

[6] C. Newport, D. Kotz, Y. Yuan, R. S. Gray, J. Liu, and C. Elliott, "Experimental evaluation of wireless simulation assumptions," SIMULATION, vol. 83, no. 9, pp. 643-661, Sep. 2007.

[7] J. Bicket, D. Aguayo, S. Biswas, and R. Morris, "Architecture and evaluation of an unplanned 802.11b mesh network," in MobiCom, 2005, pp. $31-42$.

[8] D. Aguayo, J. Bicket, S. Biswas, G. Judd, and R. Morris, "Link-level measurements from an $802.11 \mathrm{~b}$ mesh network," SIGCOMM Comput. Commun. Rev., vol. 34, no. 4, pp. 121-132, 2004.

[9] D. Reddy and G. Riley, "Measurement based physical layer modeling for wireless network simulations," in MASCOTS, 2007, pp. 46-53.

[10] P. Barsocchi, G. Oli, and F. Potorti, "Measurement-based frame error model for simulating outdoor Wi-Fi networks," IEEE Trans. Wireless. Comm., vol. 8, no. 3, pp. 1154-1158, 2009.

[11] A. Kashyap, S. Ganguly, and S. R. Das, "Measurement-based approaches for accurate simulation of 802.11-based wireless networks," in MSWIM, 2008, pp. 54-59.

[12] M. Al-Bado, C. Sengul, and R. Merz, "What details are needed for wireless simulations? - a study of a Site-Specific indoor wireless model," ser. INFOCOM, Orlando, Florida, USA, Mar. 2012.

[13] K. Pawlikowski, H. d. Joshua Jeong, and J. s. Ruth Lee, "On credibility of simulation studies of telecommunication networks," IEEE Communications Magazine, vol. 40, pp. 132-139, 2002.

[14] S. Kurkowski, T. Camp, and M. Colagrosso, "Manet simulation studies:

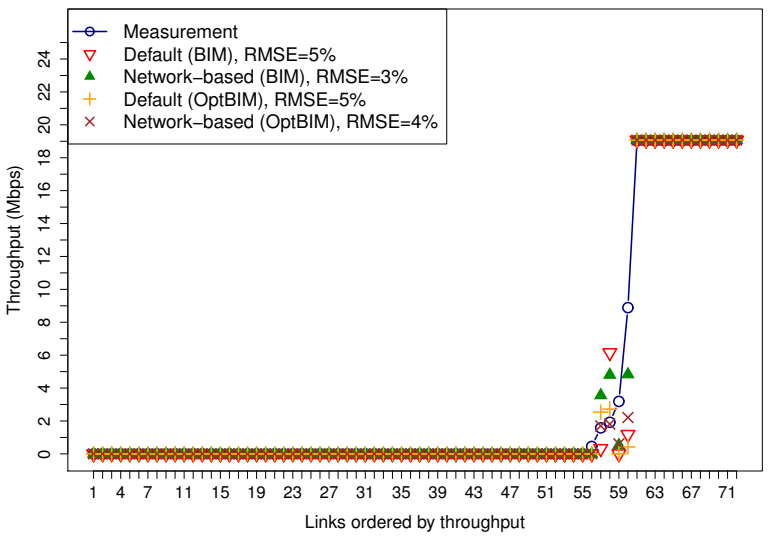

(a) UDP 54 Mbps

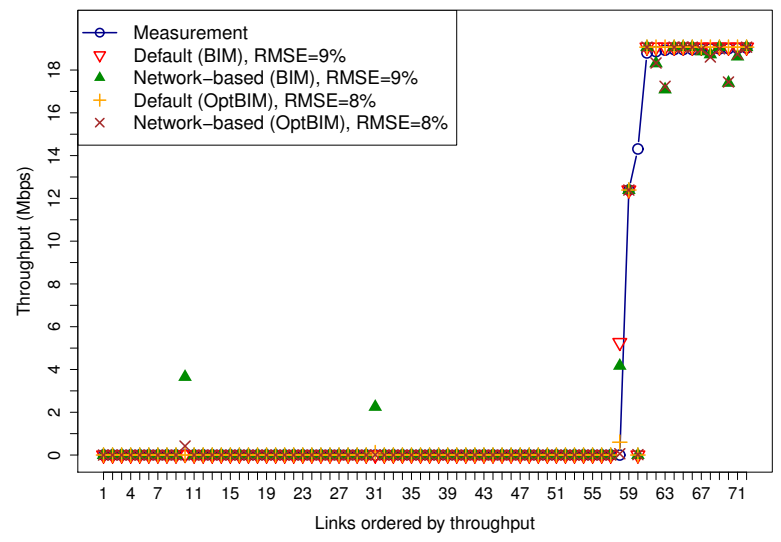

(b) TCP 54 Mbps

Fig. 5. Single flow traffic validation for $54 \mathrm{Mbps}$ data-rate.

The incredibles," SIGMOBILE Mob. Comput. Commun. Rev., vol. 9, pp. $50-61,2005$

[15] M. Takai, R. Bagrodia, K. Tang, and M. Gerla, "Efficient wireless network simulations with detailed propagation models," Wirel. Netw. vol. 7, pp. 297-305, May 2001.

[16] M. Takai, J. Martin, and R. Bagrodia, "Effects of wireless physical layer modeling in mobile ad hoc networks," ser. MobiHoc '01, 2001, pp. 8794.

[17] C. Reis, R. Mahajan, M. Rodrig, D. Wetherall, and J. Zahorjan, "Measurement-based models of delivery and interference in static wireless networks," in ACM SIGCOMM, 2006, pp. 51-62.

[18] R. Merz, H. Schiöberg, and C. Sengul, "Design of a configurable wireless network testbed with live traffic," in TridentCom, May 2010.

[19] "Atheros," http://www.wifi-stock.com/file/cm9_user_manual.pdf.

[20] "IEEE standard for information technology-telecommunications and information exchange between systems-local and metropolitan area networks-specific requirements - Part 11: wireless lan medium access control (MAC) and physical layer (PHY) specifications," pp. C1 -1184, June 2007.

[21] P. J. Husted, Y. Huanchun, and S. Aman, "Adaptive interference immunity control," US Patent 7349503, Atheros Communications, Inc., March 2008.

[22] I. Tinnirello, D. Giustiniano, L. Scalia, and G. Bianchi, "On the sideeffects of proprietary solutions for fading and interference mitigation IEEE 802.11b/g outdoor links," Computer Networks, Feb. 2009.

[23] "Bowl indoor model in ns-3," http://code.nsnam.org/rmerz/ns-3-bimsimutools2011. 